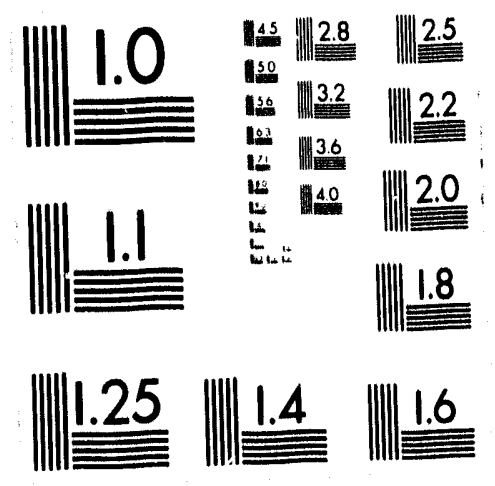



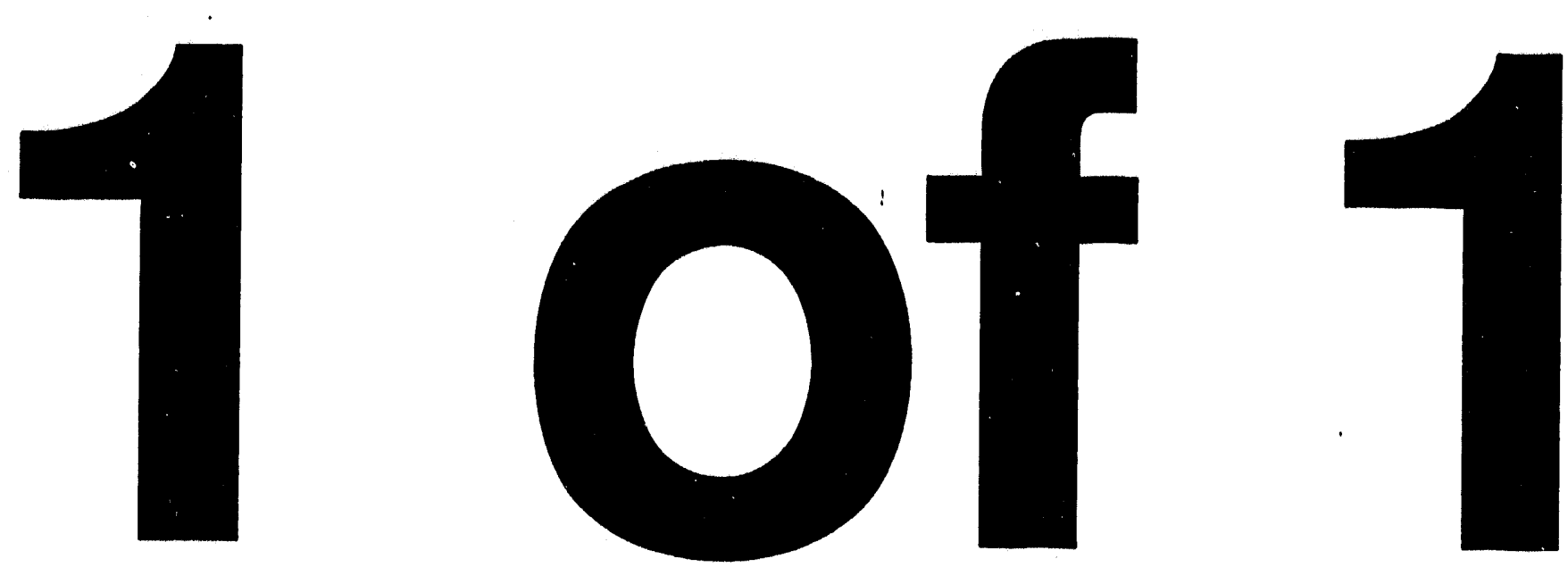


\author{
M. Larsson, G. Sundström, M. Carlson \\ Physics Department I, The Royal Institute of Technology (KTH), S-100 44 \\ Stockholm, Sweden
}

H. Danared, A. Källberg, K.G. Rensfelt, M. af Ugglas,

L. Broström, S. Mannervik, P. Sigray

Manne Siegbahn Institute of Physics, S-104 05 Stockholm, Sweden

A. Filevich

Physics Department, CNEA, Tandar, 1429 Buenos Aires, Argentina

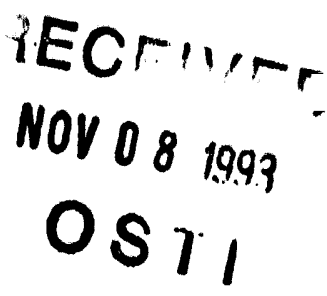

\title{
S. Datz
}

Physics Division, Oak Ridge National Laboratory, P.O. Box 2008, Oak Ridge, Tennessee 37831, USA

J.R. Mowat

Department of Physics, North Carolina State University, Raleigh, North Carolina 27695-8202, USA

\begin{abstract}
Ion storage rings offer several advantages for studies of recombination of molecular ions by electrons, a process which in general leads to dissociation. During the last year dissociative recombination of $\mathrm{H}_{3}{ }^{+}, \mathrm{HD}^{+}, \mathrm{H}_{2}{ }^{+}, \mathrm{D}_{2}{ }^{+},{ }^{3} \mathrm{HeH}^{+},{ }^{4} \mathrm{HeH}^{+}$and ${ }^{4} \mathrm{HeD}$ have been studied in storage rings in Stockholm, Heidelberg, Tokyo and Aarhus. Only a fraction of the results obtained at these places have been processed to the point where they have been published in the literature. Hence, the discussion of some of the results in this article must be regarded as tentative.
\end{abstract}

\section{INTRODUCTION}

The capture of a free electron by a molecule may be regarded as an elementary step in the general field of electron transfer reactions. If the molecule is neutral, the process leads to the formation of a temporary negative ion the fate of which depends on the electron affinity of the molecule. One possible outcome is unimolecular decomposition of the negative molecular ion into one neutral and one negatively charged fragment; this process is called dissociative attachment. If the free electron is captured by a positively charged molecular ion, the process is called dissociative recombination, the term "dissociative" being added in order to emphasize that the capture normally is followed by a unimolecular decomposition of the neutralized molecule into neutral (or, however less likely, positively and negatively charged) fragments. The process can thus be described as $\mathrm{AB}^{+}+\mathrm{e} \rightarrow \mathrm{A}+\mathrm{B}$.

\section{MASTER}


There is a fundamental difference between dissociative attachment and dissociarive recombination: attachment of a free electron to a neutral molecule is a direct process which only involves a transition berween the initial neutral molecule plus a tree electron and the final negative ion state. Thus, the cross section for dissociative attachment is in general negligible for very low electron kinetic energies (say $\ll 1 \mathrm{eV}$ ) and peaks at $1-10 \mathrm{eV}$ (roughly speaking). Dissociative recombination, on the other hand, may occur via capture into predissociating Rydberg states of the neutral molecule, or through a direct transition to a repulsive doubly excited state. The cross section may have structures, similar to those for dissociative attachment, at several $\mathrm{eV}$, but at low energies the cross section scales as $1 / E^{n}$, where $E$ is the collision energy and $n$ is a number close to unity. This fact complicates the experimental study of dissociative recombination enormously compared to the study of dissociative attachment. Likewise, the competition between different dissociation pathways at low energies complicates a theoretical treatment of the process. A further complication with recombination studies is that it is very difficult to prepare a molecular ion in a well defined quantum state. The approach to an experimental access to low energy dissociative electron-ion collisions has been the merged-beam technique. ${ }^{1}$ In this paper we will describe the most recent development of the merged-beam technique for sudies of dissociative recombination: the use of beams of molecular ions stored and phase-space manipulated in heavy-ion cooler rings. The first results from dissociative recombination ion storage ring experiments have recently appeared in the literature. ${ }^{2-4}$

\section{BASIC ASPECTS OF DISSOCIATIVE RECOMBINATION STLDIES IN STORAGE RINGS}

A good starting point for a description of the basic aspects and main advantages of studies of dissociative recombination in a storage ring is Fig. 1, which schematically shows CRYRING in Stockholm. It should, however, be pointed out that the experiments performed at TSR in Heidelberg, TARN II in Tokyo and ASTRID in Aarhus are in practice very similar to the CRYRING experiments. The ions are produced in an

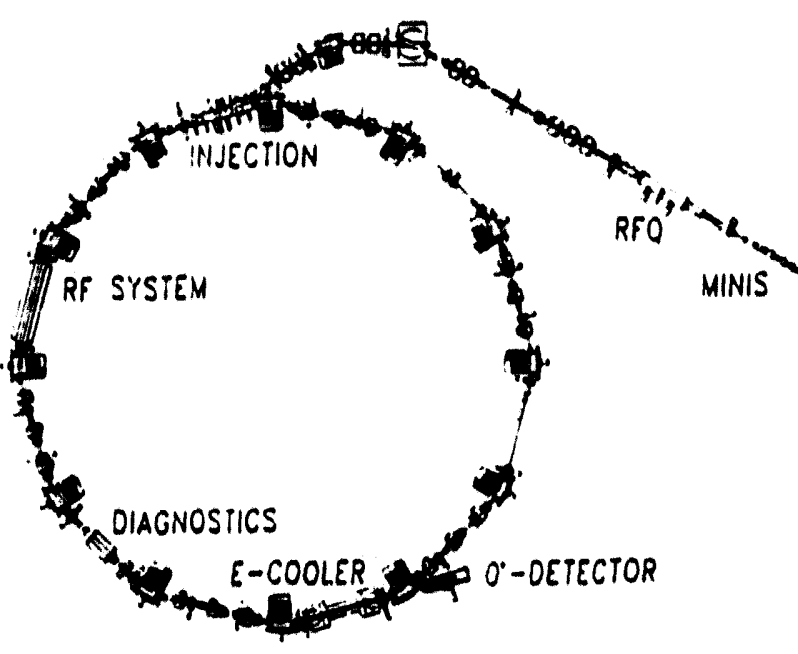

Fig. 1 Schematic diagram of CRYRING. electron-impact ion source (MINIS), extracted at $30 \mathrm{keV}$, accelerated to 300 $\mathrm{keV} / \mathrm{u}$ in a four-rod radiofrequency quadrupole (RFO) and injected into the storage ring. In CRYRING the ions are further accelerated to $96(q / M)^{2}$, where $q$ is the charge state and $M$ is the molecular mass. The acceleration to full energy takes about $2 \mathrm{~s}$. After reaching full energy the ions are cooled by a collinear beam of velocity-matched electrons (E-COOLER). For $\mathrm{H}_{3}{ }^{+}$, Schottky noise spectra showed that the longitudinal velocity spread was reduced from $8 \times 10^{-4}$ to $6 \times 10^{-5}$, which also took about $2 \mathrm{~s}$. Transverse cooling is 
expected to proceed slower, however, the cooling time has not been measured. After a variable time of a few to tens of seconds, during which cooling is continuously applied, the electron beam energy is switched from $E_{\text {cool }}$, for which the electrons are velocity matched with the ions, to $E_{\text {mese }} \sim\left(\sqrt{ } E_{\text {cool }}+\sqrt{ } E_{\text {ret }}\right)^{2}$, where $E_{\text {rol }}$ is the value of the relative collision energy for which the cross section is to be determined and $E_{\text {mes }}$ is the energy applied to the electron cooler. In order to avoid electron drag force and heating of the ion beam, the electron cooler is only kept at $E_{\text {mes }}$ for $0.3-0.5 \mathrm{~s}$ and then switched back to $E_{\text {cool }}$. During the $E_{\text {mes }}$ phase, the number of neutral particles produced in the electroncooler section are counted by an ion implanted silicon detector $\left(0^{\circ}\right.$-DETECTOR in Fig. 1). The switching between $E_{\text {cool }}$ and $E_{\text {mes }}$ is repeated a couple of times per injection cycle. Owing to collisions in the rest gas, which is kept at a vacuum in the low $10^{-11}$ torr region, the stored beam is eventually destroyed and after 10-30 s the storage ring is filled with new ions. In order to obtain absolute cross sections, the current of the circulating beam is measured by a current transformer.

The procedure described above offers the following advantages for measurements of dissociative recombination:

i) The ion beam is circulated in the storage ring so that each ion is passing the electron target of the order of a million times per second. This greatly increases the effective current. Combined with the high electron beam current provided by a cooler designed for high ion beam velocities, ion storage ring technology provides a large enhancement of luminosity compared to single-pass experiments.

ii) Phase-space cooling of the stored molecules reduces the energy spread in the ion beam to the point where it can be neglected when cross sections are extracted. The cooling process also aligns the ion beam with respect to the electron beam, thus minimizing deterioration of the resolution due to beam misalignment.

iii) Ion storage facilitates relaxation of excited vibrational levels in infrared active molecules (i.e. essentially all molecules except homonuclear) so that experiments can be performed with ions populating only their vibrational ground state. This may be the single most important advantage of the ring technique for handling molecular ions.

iv) By performing the experiments at high energies, processes like $\mathrm{AB}^{+}+\mathrm{Rg} \rightarrow \mathrm{A}+\mathrm{B}$ ( $\mathrm{Rg}$ represents a rest gas atom) are strongly suppressed. In essence this means that the dissociative recombination data can be recorded against a zero background.

The rate of the reaction $\mathrm{AB}^{+}+\mathrm{e} \rightarrow \mathrm{A}+\mathrm{B}$ in the storage ring is given by the relation $R=p_{0} v_{\mathrm{rel}} \sigma_{\mathrm{DR}} l / L$ ion $^{-1} \mathrm{~s}^{-1}$, where $\rho_{\mathrm{o}}$ is the electron density in the merged-beam region, $v_{\text {ret }}$ is the relative collision velocity, $\sigma_{D R}$ is the dissociative recombination cross section, $l$ is the length of the interaction region ( $l=0.9 \mathrm{~m}$ for CRYRING) and $L$ is the circumference of the storage ring ( $L=51.6 \mathrm{~m}$ for CRYRING). This relation holds of course only strictly for the ideal case of monoenergetic beams. In practice, at least for very low collision energies, one must consider the deviation from monoenergetic conditions. For a cooled beam, the ions have the same temperature, and hence the same energy spread, as the electrons. Since the ions are several thousands times heavier than the electrons, the ion velocity spread can be neglected. An electron beam accelerated to several $\mathrm{keV}$ is characterized by transverse and longitudinal temperatures $T_{\perp}$ and $T_{1}$, which differ by orders of magnitude. The term $v_{\mathrm{Trt}} \sigma_{\mathrm{DR}}$ in the expression for $R$ is then replaced by $\left\langle\nu_{\mathrm{rel}} \sigma_{\mathrm{DR}}\right\rangle=\int \nu_{\mathrm{rat}} \sigma_{\mathrm{DR}}(v) f(v)$ d $v$, i.e. an average of a velocity-weighted cross 
section over a "flattened" electron velocity distribution,

$$
f(v) d v=\left(\frac{m_{e}}{k T_{\perp}}\right) \exp \left(-m_{e} v_{\perp}^{2} / 2 k T_{\perp}\right) v_{\perp} d v_{\perp}\left(\frac{m_{e}}{2 \pi k T_{1}}\right)^{1 / 2} \exp \left(-m_{e}\left(v_{1}-\Delta\right)^{2} / 2 k T_{\perp}\right) d v_{1}
$$

where $\Delta$ is the detuning velocity corresponding to $E_{\mathrm{ral}}=m_{\mathrm{o}} \Delta^{2} / 2$. The transverse electron temperature has for the CRYRING electron cooler been measured to $k T_{\perp}=0.1 \mathrm{eV}$. The longitudinal temperature is much lower owing to the acceleration cooling mechanism. For $\mathrm{H}_{3}{ }^{+}$, it was demonstrated that $\left\langle\nu_{\mathrm{rel}} \sigma_{\mathrm{DR}}>\right.$ could be replaced by the simpler expression $\nu_{\text {rel }} \sigma_{\mathrm{DR}}$ for collision energies down to $0.0025 \mathrm{eV}$ under the assumption of $T_{1}=0 .{ }^{4}$ More recently it has been demonstrated that $\left\langle\nu_{\mathrm{rol}} \sigma_{\mathrm{DR}}\right\rangle$ is insensitive to whether one uses the ideal case of $T_{1}=0$ or a more realistic value of $k T_{1}=10^{-4} \mathrm{eV}$.

The upshot of the discussion above is that cross sections for collision energies much smaller than $k T_{\perp}=0.1 \mathrm{eV}$ can readily be measured in an electron cooler. However, resonances located at energies smaller than $0.1 \mathrm{eV}$, and with widths much smaller than $0.1 \mathrm{eV}$, would be difficult to observe.

\section{SOME EXAMPLES: $\mathrm{H}_{3}^{+}$AND $\mathrm{HeH}^{+}$}

The first dissociative recombination storage ring experiments ${ }^{2-4}$ all concerned different molecules. In Tokyo (TARN II) recombination of $\mathrm{HeH}^{+}$was studied, ${ }^{2}$ in Heidelberg (TSR) the $\mathrm{HD}^{+}$molecule was chosen for a first experiment, ${ }^{3}$ and in Stockholm (CRYRING) $\mathrm{H}_{3}{ }^{+}$was the first molecule on the agenda. ${ }^{4}$ All three experiments exploited the infrared radiative cooling mechanism and were thus able to obtain results for vibrationally relaxed ions. Only the CRYRING experiment provided absolute cross sections.

The simplest polyatomic molecular ion $\mathrm{H}_{3}{ }^{+}$is very important in many natural environments. It occupies a central role in interstellar chemistry and it has recently been observed in emission in polar regions of Jupiter and very recently in Uranus and Saturn. ${ }^{6}$ The importance of $\mathrm{H}_{3}{ }^{+}$may be illustrated by the fact that it has been the subject of several review articles. ${ }^{7.9}$ The dissociative recombination rate of $\mathrm{H}_{3}{ }^{+}$has been a controversial subject for a long time. In the most recent compilation of measured rates one can notice a more than four orders of magnitude spread in the data (few times $10^{-7}$. $\left.10^{-11} \mathrm{~cm}^{3} \mathrm{~s}^{-1}\right) .{ }^{10}$ These results have been obtained by means of various techniques, such as extraction of rates from measured cross sections with the merged-beam technique, ${ }^{11}$ infrared absorption spectroscopy, ${ }^{12}$ and different types of flowing afterglow experiments. ${ }^{10,13,14}$ An additional problem has been that, despite some efforts, ${ }^{15,16}$ theory has been unable to provide any quantitative guidance.

The rate of the reaction $\mathrm{H}_{3}{ }^{+}+\mathrm{e} \rightarrow \mathrm{H}+\mathrm{H}+\mathrm{H}$ or $\mathrm{H}_{2}+\mathrm{H}$ was measured in CRYRING and converted to cross sections by the relation $R=p_{\theta} v_{\text {rel }} \sigma_{\mathrm{DR}} / / \mathrm{L}_{\mathrm{ion}} \mathrm{is}^{-1} \mathrm{~s}^{-1}$. The measurements were carried out for collision energies $E_{\text {rel }}$ in the range $0.0025-30 \mathrm{eV}$. The results are shown in Fig. 2. 


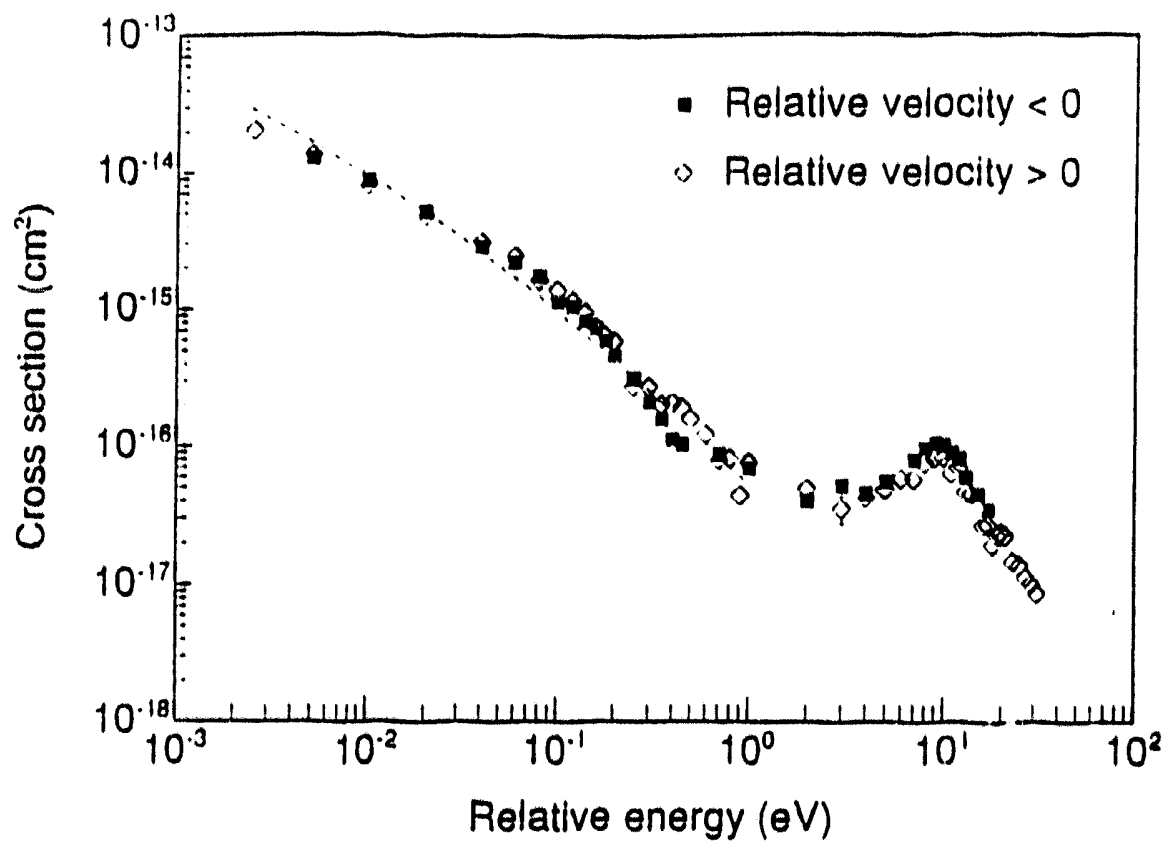

Fig. 2. Total cross sections for forming $\mathrm{H}+\mathrm{H}+\mathrm{H}$ and $\mathrm{H}+\mathrm{H}_{2}$. Diamonds illustrate the results obtained by letting the electrons travel faster than the ions (denoted "Relative velocity >0"), and filled squares illustrate the results obtained by letting the electrons travel slower than the ions (denoted "Relative velocity < 0 ").

The low energy part of the cross section behaves as expected, i.e. it displays a $1 / E^{n}$ dependence. At high energy, the cross section increases and peaks at $9.5 \mathrm{eV}$. This peak represents the direct, resonance channel of the dissociative recombination process in $\mathrm{H}_{3}{ }^{+}$. Simple model calculations employing potential curves of $\mathrm{H}_{3}{ }^{+}$and $\mathrm{H}_{3}$ show that the shape of the $9.5 \mathrm{eV}$ peak indicates that the cross sections were obtained for vibrationally relaxed ions. The data were taken about $10 \mathrm{~s}$ after the $\mathrm{H}_{3}{ }^{+}$ions were produced in the ion source MINIS. This time scale is sufficient to allow a full vibrational relaxation, as follows from $a b$ initio calculations of transition probabilities in $\mathrm{H}_{3}{ }^{+17}$ The observation of the direct channel at $9.5 \mathrm{eV}$ has stimulated theoretical calculations of this process. ${ }^{18}$

The expected cross section behaviour for crossings of neutral dissociative states with a bound ion state has been investigated by model calculations. ${ }^{19}$ The quantitative agreement between the measured cross sections in Fig. 2 and the one calculated in ref. 19 (case $B$ crossing) is amusing, but of course purely accidental. In $\mathrm{H}_{3}{ }^{+}$the potential crossing occurs at an energy corresponding to vibrational quantum number $\nu=3$ (as compared to $v=1$ in ref. 19) and hence one would expect a much smaller cross section at low energy than shown in Fig. 2. In fact, the absence of a favourable potential crossing for vibrationally cool $\mathrm{H}_{3}{ }^{+}$has been advanced as an argument for a low recombination rate for $\mathrm{H}_{3}{ }^{+}(v=0)$, and for a strongly vibrationally dependent recombination rate. ${ }^{13,16}$ In order to facilitate a direct comparison between our measured cross sections and directly measured dissociative recombination rates, ${ }^{10.12 .14}$ the rate coefficient must be extracted from the measured cross sections via 


$$
\alpha(T)=\int \frac{8 \pi m_{e} E}{\left(2 \pi m_{\rho} k T\right)^{3 / 2}} \sigma(E) \exp (-E / k T) d E
$$

The integral is sensitive to the cross sections at very low collision energies, and for this reason we have carried out measurements in the region $0.00005-0.0025 \mathrm{eV}$. The data indicate a $1 / \mathrm{E}^{1.2}$ cross section dependence and favour a recombination rate of the order of $10^{-7} \mathrm{~cm}^{3} \mathrm{~s}^{-1}$ at $300 \mathrm{~K}$. These results are preliminary; the final results will be given in a forthcoming publication. It would seem like a $10^{-7} \mathrm{~cm}^{3} \mathrm{~s}^{-1}$ rate is irreconcilable with theory; ${ }^{16}$ however, a new, multistep recombination mechanism for $\mathrm{H}_{3}{ }^{+}$has recently been proposed ${ }^{19}$ which seems to accommodate a high recombination rate. Finally the claim for a very low rate $\left(-10^{-11} \mathrm{~cm}^{3} \mathrm{~s}^{-1}\right),{ }^{13}$ obtained with the flowing afterglow/Langmuir probe method, has been withdrawn. New measurements give $(1-2) \times 10^{-8} \mathrm{~cm}^{3} \mathrm{~s}^{-1}$ at $300 \mathrm{~K}^{20}$ which is still low, but not in a drastic disagreement with the storage ring result. The $\mathrm{H}_{3}{ }^{+}$ dissociative recombination story has taken a big leap forward during the last year.

The ground state potential curve of $\mathrm{HeH}^{+}$is not crossed by any neutral state, and for this reason it was for a long time assumed that the recombination cross section for this molecule should be very small. The first merged-beam results ${ }^{21}$ for $\mathrm{HeH}^{+}$therefore came as something of a surprise; the cross section was much larger than one would have expected from a study of the relevant potential curves. Efforts to understand the mechanism were unsuccessful, ${ }^{22}$ and it was for some time speculated whether the merged-beam results could have been affected by the presence of excited triplet state population in the ion beam. ${ }^{21}$ This possibility was effectively refuted when the final results were published; measurements of dissociative excitation cross sections were used to demonstrate that, under certain conditions, the $\mathrm{HeH}^{+}$ions did not populate excited states. $^{23}$

$\mathrm{HeH}^{+}$was chosen as the first molecule for a dissociative recombination experiment at TARN II, ${ }^{2}$ possibly because $\mathrm{HeH}^{+}$is known to cool radiatively on a time scale amenable for storage ring experiments. ${ }^{24} \mathrm{~A}$ previously unobserved peak in the cross section at $20 \mathrm{eV}$ was found and explained as dissociation through two-electron excited states which form Rydberg manifolds converging to excited states of $\mathrm{HeH}^{+}$. Similar high-energy structures were also found for $\mathrm{HD}^{+}$in the Heidelberg-experiment. ${ }^{3}$ Only relative cross sections were obtained in the TARN II-experiment, and the resolution at low energy was insufficient to reveal any structures. Recently absolute cross sections were measured for ${ }^{3} \mathrm{HeH}^{+}$in CRYRING. The preliminary results are shown in Fig. 3. The $20 \mathrm{eV}$ peak is observed and there is an additional peak close to $30 \mathrm{eV}$ which is discernible also in the TARN II-data. ${ }^{2}$ The cross section at low energy is smaller than the single-pass merged-beam result, ${ }^{23}$ however, the difference is within a factor of five. The question is now whether there is a suitable mechanism which could account for a large cross section despite the absence of a potential crossing. A hint is contained in the experiments with charge exchange between $\mathrm{HeH}^{+}$and $\mathrm{Cs}^{25.26}$ These experiments show 
that Rydberg states of $\mathrm{HeH}$ converging to the $\mathrm{HeH}^{+}$ground state predissociate by coupling to the repulsive ground state of $\mathrm{HeH}^{27} \mathrm{Ab}$ initio calculations of elastic

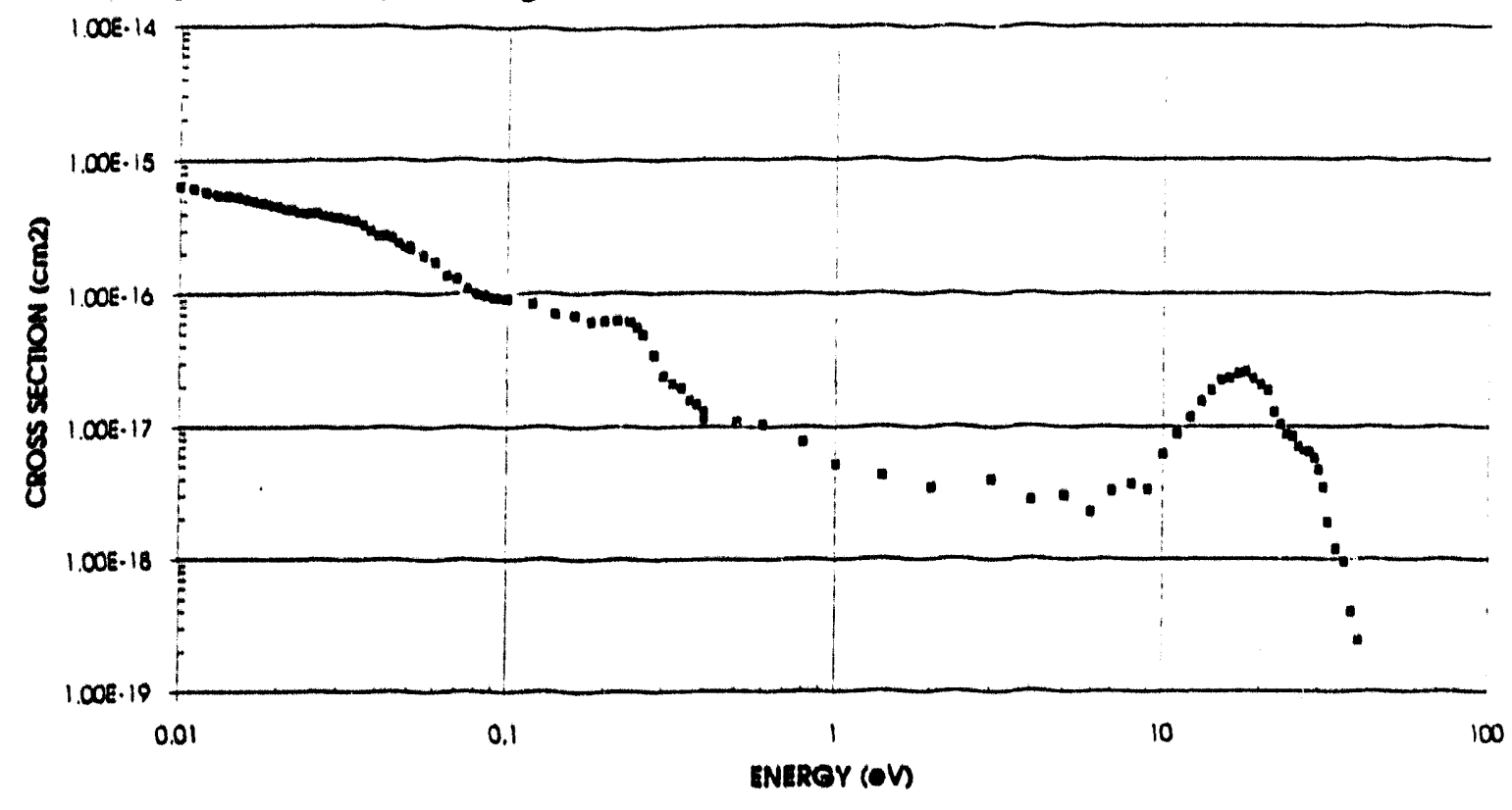

Fig. 3. Preliminary cross sections for the process ${ }^{3} \mathrm{HeH}^{+}+\mathrm{e} \rightarrow{ }^{3} \mathrm{He}+\mathrm{H}$.

scattering cross sections for electron $\mathrm{HeH}^{+}$collision $\mathrm{s}^{28}$ have further indicated a breakdown of the Bom-Oppenheimer approximation at low collision energies, a prerequisite for large recombination cross section. Very recently, calculations of dissociative recombination cross sections for $\mathrm{HeH}^{+}$have provided compelling evidence that a large recombination rate indeed can be obtained also in the non-crossing case. ${ }^{29,30}$ In view of this theoretical development, the recent claim that measured recombination at low energy is due solely to the presence of triplet state popularion in the ion beam appears the more puzzling.

Ion storage rings have proved to be very powerful for studies of dissociative recombination of molecular ions, and it seems that the development of this new experimental tool has been stimulating for theorists. The full potential of the storage rings has not yet been exploited and an exciting future can be anticipated.

\section{ACKNOWLEDGEMENTS}

We are indebted to L.H. Andersen, D.R. Bates, A. Dalgarno, S.L. Guberman, D. Habs, K.C. Kulander, D. Smith. T. Tanabe and J. Tennyson for valuable correspondence and discussions, preprints and reprints.

\section{REFERENCES}

1. J.B.A. Mitchell, Phys. Rep. 186, 215 (1990).

2. T. Tanabe, I. Katayama, N. Inoue, K. Chida, Y. Araki, T. Watanabe, M.Yoshizawa, S. Ohtani, and K. Noda, Phys. Rev. Lett. 70, 422 (1993). 
3 P. Forck, M. Grieser, D. Habs, A. Lampert, R. Repnow, D. Schwalm, A. Wolf, D. Zajfman, Phys. Rev. Lett. 70, 426 (1993).

4. M. Larsson, H. Danared, J.R. Mowat, P. Sigray, G. Sundström, L. Broström, A. Filevich, A. Kallberg, S. Mannervik, K.G. Rensfelt, and S. Datz, Phys. Rev. Lett. 70, 430 (1993).

5. R.J. Mowat (to be published).

6. T. Oka and M.-F. Jagod, J. Chem. Soc. Faraday Trans, to be published in 89, (1993).

7. T. Oka, in Molecular lons: Spectroscopy, Structure and Chemistry, edited by T.A. Miller and V.E. Bondybey (North-Holland, New York, 1983), p. 73.

8. T. Oka, Rev. Mod. Phys. 64, 1141 (1992).

9. A. Dalgarno (to be published).

10. A. Canosa, J.C. Gomet, B.R. Rowe, J.B.A. Mitchell, and J.L. Queffelec, J. Chem. Phys. 97, 1028 (1992).

11. H. Hus, F.B. Yousif, A. Sen, and J.B.A. Mitchell, Phys. Rev. A 38, 658 (1988).

12. T. Amano, J. Chem. Phys. 92,6492 (1990).

13. N.G. Adams and D. Smith, in Dissociative Recombination: Theory, Experiment and Applications, edited by J.B.A. Mitchell and S.L. Guberman (World Scientific, Singapore, 1989), p. 124.

14. The reader is referred to ref. 10 for a complete list of dissociative recomination results for $\mathrm{H}_{3}{ }^{+}$.

15. K.C. Kulander and M.F. Guest, J. Phys. B 12, L501 (1979).

16. H.H. Michels and R.H. Hobbs, Astrophys. J. 286, L27 (1984).

17. B.M. Dinelli, S. Miller, and J. Tennyson, J. Mol. Spectrosc. 153, 718 (1992).

18. K.C. Kulander and A.E. Orel (private communication).

19. D.R. Bates, M.F. Guest, and R.A. Kendall, Planet. Space Sci. 41, 9 (1993).

20. D. Smith and P. Spanel (preprint, 1993).

21. J.B.A. Mitchell and F.B. Yousidf, in Dissociative Recombination: Theory, Experiment and Applications, edited by J.B.A. Mitchell and S.L. Guberman (World Scientific, Singapore, 1989), p. 109.

22. H.H. Michels, in Dissociative Recombination: Theory, Experiment and Applications, edited by J.B.A. Mitchell and S.L. Guberman (World Scientific, Singapore, 1989), p. 97.

23. F.B. Yousif and J.B.A. Mitchell, Phys. Rev. A 40, 4318 (1989).

24. S. Datz and M. Larsson, Phys. Scripta 46, 343 (1992).

25. W.J. van der Zande, W. Koot, D.P. de Bruijn, and C. Kubach, Phys. Rev. Lett. 57, 1219 (1986).

26. J.R. Peterson and Y.K. Bae, Phys. Rev. A 34, 3517 (1986).

27. A.B. Alekseyev, V.S. Ivanov, A.M. Paravilov, and O.D. Shestakov, Chem. Phys. 155, 173 (1991).

28. B.K. Sarpal, J. Tennyson, and L.M. Morgan, J. Phys. B 24, 1851 (1991)

29. S.L. Guberman (private communication).

30. J. Tennyson (private communication).

31. J.B.A. Mitchell, M. Chibisov, and F.B. Yousif, DAMOP meeting, TC 2 (1993). 


\section{DISCLAIMER}

This report was prepared as an account of work sponsored by an agency of the Unitod States Government. Neither the United States Government nor any agency thereof, nor any of their omployees, makes any warranty, express or impliod, or assumes any logal liability or responsibility for the accuracy, completeness, or usefulness of any information, apparatus, product, or process disclosed, or represents that its use would not infringe privately owned rights. Reference herein to any specific commercial product, process, or service by trade name, trademark, manufacturer, or otherwise does not necessarily constitute or imply its endorsement, recommendation, or favoring by the United States Government or any agency thereof. The views and opinions of authors expressed herein do not necessarily state or reflect those of the United States Government or any agency thereof. 


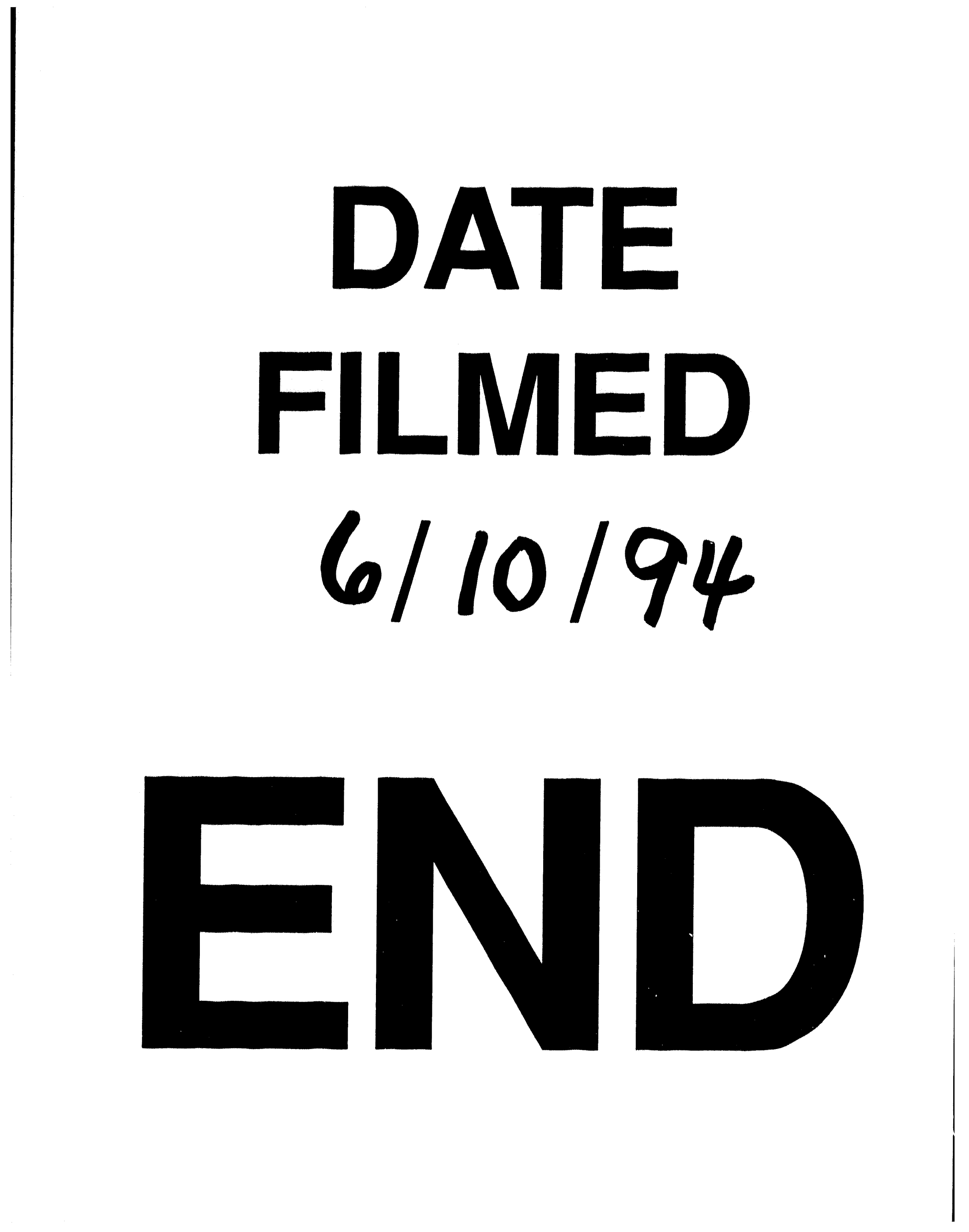


